\title{
En coup de sabre presenting as status epilepticus
}

\author{
Pawan Kashyape $^{1}$ (D) $\cdot$ Ajay Prashanth D'Souza ${ }^{2} \cdot$ Basil Fathalla $^{3}$
}

Received: 3 June 2020 /Revised: 6 July 2020 / Accepted: 9 July 2020 / Published online: 18 July 2020

(C) International League of Associations for Rheumatology (ILAR) 2020

\section{Presentation}

A healthy 10-year-old male presented with the first episode of unprovoked status epilepticus with focal onset and recovered with mild right side hemiparesis.

CT scan showed scattered multifocal calcified lesions in the left cerebral parenchyma (Fig. 1d).

Initial radiological diagnoses included latent toxoplasmosis or neurocysticercosis.

On follow-up visit, an induration and linear alopecia were noticed involving the forehead and scalp ipsilateral to the intracranial lesions (Fig. 1a, c), which had been long-standing but previously not recognized as being relevant.

MRI brain was obtained (Fig. 1b), which showed linear streaky white matter high signal, cortical gliosis, and focal thinning of the skull bone ipsilateral to the cutaneous lesions suggesting a diagnosis of linear scleroderma with neurological involvement. The lesion was confirmed on histopathology by doing a scalp biopsy. The patient had no evidence of Raynaud's phenomenon, sclerodactyly, or any other organ involvement. No systemic symptoms were reported. Antinuclear antibodies were absent. The patient was managed using anticonvulsants and immune modulation.

Pawan Kashyape

pawankashyape@yahoo.co.uk

1 Department of Pediatric Neurology, Latifa Women and Children Hospital, Dubai, United Arab Emirates

2 Department of Radiology, Al Jalila Children's Hospital, Dubai, United Arab Emirates

3 Department of Pediatric Rheumatology, Al Jalila Children's Hospital, Dubai, United Arab Emirates

\section{Discussion}

Scleroderma is a chronic autoimmune connective tissue disease characterized by inflammation and excess collagen deposition in skin and internal organs, vascular injury, and fibrosis. It can be localized (also known as morphea) or systemic with internal organ involvement.

The localized and systemic subtypes are mainly distinguished based on absence of Raynaud's phenomenon, autoantibodies, and nailfold capillary changes as well as visceral involvement in the former [1].

Localized scleroderma has equal prevalence in adults and children and is considered a distinct disease entity from systemic sclerosis [1].

The linear form of localized scleroderma is most common in children.

Localized scleroderma is not an exclusively cutaneous disease, however, with occasional evidence of internal organ involvement and even evolution into the systemic subtype especially reported in pediatric patients [1,2].

The linear form of localized scleroderma involving the head is known as en coup de sabre (ECDS). The prevalence is less than 3 per 100,000 .

It has increased risk of involving the underlying central nervous system and the eye [1].

Neurological involvement is rare and can present with a myriad of syndromes including epilepsy, headache, focal deficits, movement disorder, trigeminal neuralgia, progressive cerebral or cerebellar atrophy, and Rasmussen syndrome [3, 4].

The scalp lesion is usually ipsilateral to the CNS lesions, and this along with thinning of the cortical skull bone should lead to the correct diagnosis [3].

All patients with morphea involving the head and neck should be closely monitored for central nervous system and ophthalmological complications.

In our patient, the combination of focal seizure, unilateral scalp, and forehead cutaneous lesions and ipsilateral positive MRI findings point towards a likely causal association between en coup de sabre and the status epilepticus. 
Fig. 1 a Left scalp linear alopecia. b MRI brain showing linear streaky white matter high signal, cortical gliosis, and focal thinning of the overlying skull bone on the left hemisphere. $\mathbf{c}$ Left eyebrow and forehead induration. d CT scan brain showing multifocal calcifications in the left cerebral hemisphere

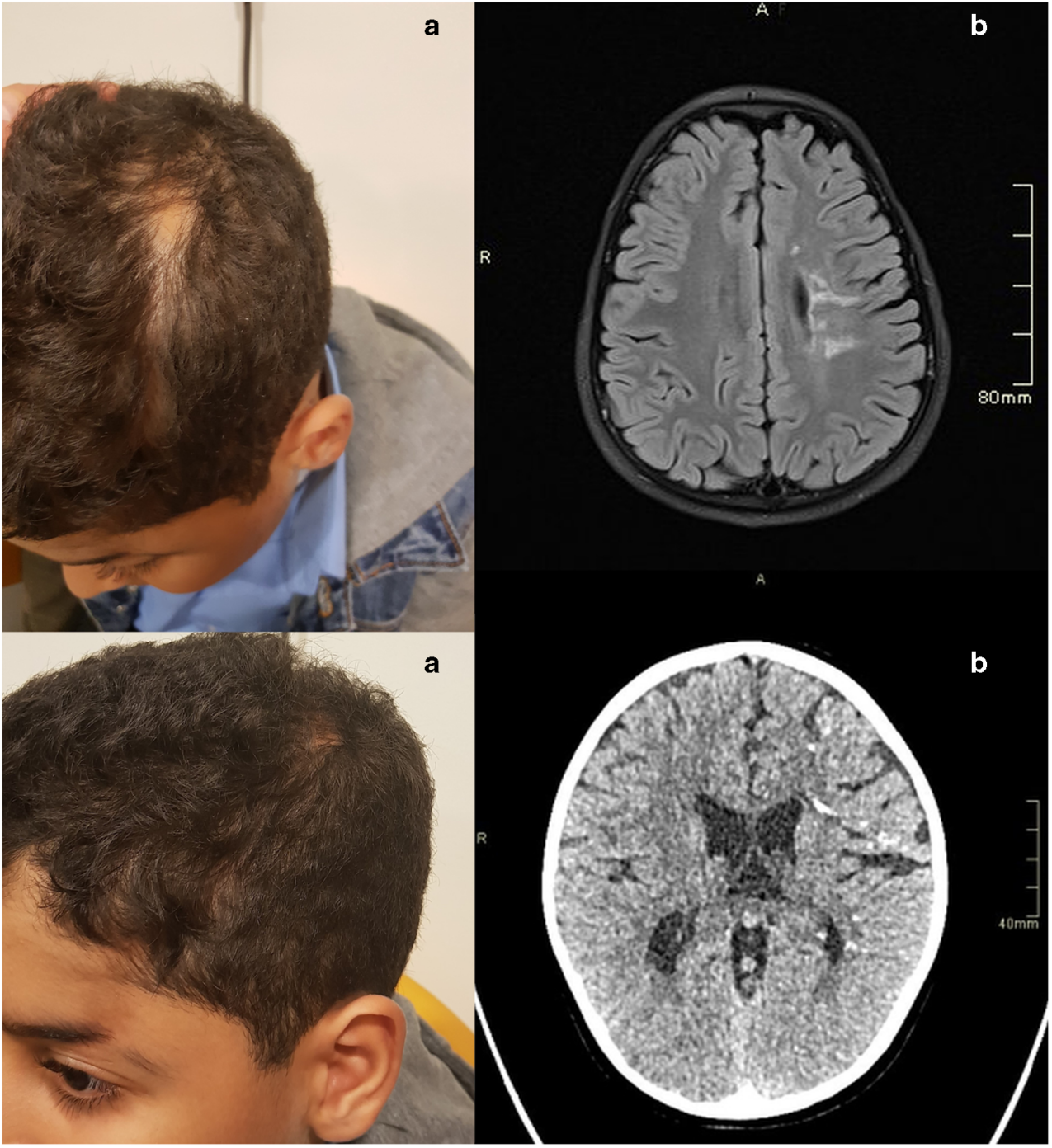

\section{Compliance with ethical standards}

Disclosures None.

Informed consent Written and informed consent was taken from the patient's parent for publication of the figure.

\section{References}

1. Fett N, Werth V (2011) Update on morphea: part I. epidemiology, clinical presentation, and pathogenesis. J Am Acad Dermatol 64(2): 217-228
2. Birdi N, Laxer R, Thorner P, Fritzler M, Silverman E (1993) Localized scleroderma progressing to systemic disease. Case report and review of the literature. Arthritis Rheum 36(3):410-415

3. Kister I, Inglese M, Laxer R, Herbert J (2008) Neurologic manifestations of localized scleroderma. Neurol 71:1538-1545

4. Amaral TN, Peres FA, Lapa AT, Marques-Neto J, Appenzeller S (2013) Neurologic involvement in scleroderma: a systematic review. Semin Arthritis Rheum 43:335-347

Publisher's note Springer Nature remains neutral with regard to jurisdictional claims in published maps and institutional affiliations. 